\title{
Breast Magnetic Resonance Imaging of Multicentric, Multifocal and Bilateral Cancer - A Case-based Review
}

\author{
Virginia Pérez Dueñas, ${ }^{1}$ María Ruíz de Gopegui Andreu, ${ }^{2}$ Sara Morón Hodge ${ }^{3}$ and \\ Asunción Suárez Manrique ${ }^{4}$
}

\begin{abstract}
1. Radiology Consultant, Department of Radiology, Hospital Universitario Madrid Sanchinarro; 2. Radiology Consultant, Department of Radiology, Hospital Infanta Elena, Hospital Universitario La Paz; 3. Doctor, Hospital Universitario La Paz; 4. Pathology Consultant, Hospital Universitario La Paz
\end{abstract}

\section{Abstract}

Multifocal or multicentric breast cancer can be difficult to detect on mammography or ultrasound, particularly in patients with dense breast tissue. A multimodality approach that includes breast magnetic resonance imaging (MRI) is indicated, particularly when conservative surgery is being considered as it is the most sensitive technique for identifying additional sites of disease. However, its influence on recurrence and survival rates has yet not been clearly established, and false-positive cases may lead to more aggressive management and treatment. Radiologists should therefore be aware of relevant breast MRI findings. Infiltrating carcinomas, contralateral unsuspected carcinomas, occult carcinomas, false-positive cases and post-chemotherapy changes. Several cases of multiple-site breast carcinomas and their corresponding mammographic, ultrasound and MRI features have been reviewed for this article, in which the definition and differences between multifocal, multicentric and contralateral breast carcinoma are explained and the most relevant imaging findings on MRI are illustrated and correlated with mammogram and ultrasound findings. Finally, the role of breast MRI in the pre-operative assessment of breast cancer is discussed.
\end{abstract}

\section{Keywords}

Breast neoplasm, invasive lobular/ductal carcinoma, multifocal/multicentric extent, magnetic resonance imaging, pre-operative staging, false-positive, local recurrence rate, survival rate

Disclosure: The authors have no conflicts of interest to declare.

Received: 3 July 2010 Accepted: 23 January 2011 Citation: European Oncology \& Haematology, 2011;7(1):24-30 DOI: 10.17925/EOH.2011.07.01.24

Correspondence: Virginia Pérez Dueñas, Department of Radiology, Hospital Universitario Madrid Sanchinarro, C/ Oña 13, 28050 Madrid, Spain. E: virpedue@gmail.com

Breast cancer is an important public health problem, as it is the leading cause of death from cancer in women and the leading cause of death in 35-55-year-old women in the EU. Well-known risk factors are a previous breast carcinoma, atypical ductal hyperplasia, atypical columnar hyperplasia, lobular lesion in situ, papillomatosis or atypical papillary lesion, mediastinal radiotherapy and family history of breast cancer, especially the positive genes BRCA 1 and $2 .{ }^{1}$

Invasive breast carcinoma includes a wide range of tumours. The most frequent is invasive ductal carcinoma not otherwise specified ([NOS] 60-80\%), followed by invasive lobular carcinoma (about 15\%), which is often multicentric or bilateral. The remaining most frequent subtypes are medullary, mucinous, papillary and tubular carcinomas, each of which occurs with a frequency of approximately $2-4 \%$.

It is important to establish the classification of breast cancer according to its local extent. Multifocal carcinoma refers to two or more tumour areas in a unique quadrant or a distance of $<4-5 \mathrm{~cm}$ (although in breasts of small volume it can involve several quadrants, see Figure 1). Multicentric carcinoma refers to two or more tumour areas in different quadrants of the same breast/to a distance $>4-5 \mathrm{~cm}$ (see Figures 2-3). Contralateral cancer can be synchronous, when the detection of a contralateral tumour occurs in the first six months following diagnosis of the primary tumour, or metachronous, when the recurrence is later. ${ }^{1}$ Multifocal or multicentric carcinomas are more frequent in young patients or peri-menopausal women with large tumours ( $>5 \mathrm{~cm}$ ) and high-density fibroglandular parenchyma, women with a family history of breast cancer and in cases of invasive lobular carcinoma.

\section{Imaging Techniques in Breast Cancer}

Breast cancer detection requires a multimodality approach and several imaging modalities must be adequately employed by the radiologist. For this reason we will include a short review of mammography and breast ultrasounds findings and indications before explaining the role of breast magnetic resonance imaging (MRI).

\section{Mammography}

Mammography is the primary diagnostic imaging modality in the evaluation of any mammary pathology because it is accessible, rapid, reproducible, relatively cheap and useful. The sensitivity and specificity for screening of breast cancer depend on the quality of the images, the experience of the radiologist and the reason for the imaging: screening versus diagnostic mammogram in symptomatic patients. It has been demonstrated that the likelihood of death from breast cancer is $50 \%$ lower in women who regularly participate in screening mammography programmes than in women who do not, with a similar death rate in the latter group to those obtained prior to 
Figure 1: Multifocal Carcinoma in a 37-year-old Woman with Three Palpable Nodules in the Right Breast
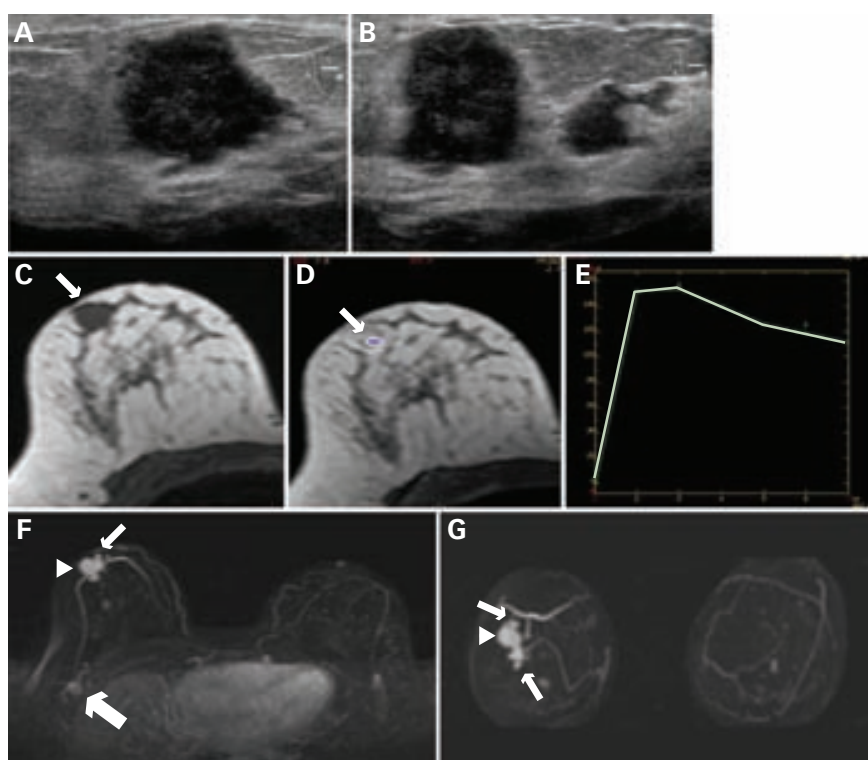

A and B: Radial ultrasound image shows three hypoechoic masses with internal echoes, craniocaudal diameter greater than the mediolateral diameter and microlobulated contours - features suggestive of malignancy. Fine-needle aspiration cytology (FNAC) was performed and all of the masses were positive for malignancy: poorly differentiated (G3) infiltrative ductal carcinoma with necrosis and intraductal component, all in the same quadrant. C-G: Magnetic resonance imaging (MRI) of both breasts was performed for detecting additional foci and pre-surgical staging. Unenhanced (C) and enhanced MRI (D) show a hypointense mass with peripheral enhancement that presents a type III enhancement curve (E). Axial (F) and coronal (G) maximum-intensity projection (MIP) reconstructions show the enhancing mass (arrowhead) in the upper lateral quadrant of the right breast, with irregular shape, lobulated margins and several satellite lesions (arrows). A round and enhancing right axillary lymphadenopathy is also seen (thick arrow). The treatment decided on was right mastectomy.

the introduction of screening mammograms. ${ }^{2}$ The sensitivity of mammography is higher in fatty breasts and decreases in dense breasts, especially in young women. Sensitivity for the detection of multifocal-multicentric carcinoma is $66 \%{ }^{3}$ The classic signs of malignancy in mammography are spiculated nodule, irregular shape, microlobulated or blurred margins and a group of amorphous, heterogeneous or pleomorphic microcalcifications. ${ }^{4}$

\section{Breast Ultrasound}

Sonography is the second most important imaging modality for the detection of mammary pathology. It is indicated in young women who have a very dense parenchyma during pregnancy and breastfeeding, because the fibroglandular component of the parenchyma grows; in inflammatory pathology, because it is better tolerated and identifies abscesses better than mammography; and in mastectomised women. It is also indicated as a complementary technique after mammography for the study of the breast with prosthesis, unspecific findings in mammogram or characterisation of injuries that cannot be completely purified by means of mammography, nipple secretion, negative mammography and palpable tumour, probable breast metastatic disease or women with high-risk factors and dense breasts. ${ }^{5}$ Finally, ultrasound is an excellent guide for interventional procedures; it is diagnostic for cysts and allows suitable definition of benign nodules that do not need biopsies. ${ }^{6}$ Breast ultrasound is not recommended as a screening technique mainly because of three reasons: microcalcifications are very difficult to identify, it depends very much on the sonographer experience and the procedure takes a long time. Nevertheless, ultrasound can detect lesions in cases where the mammogram is absolutely normal or shows
Figure 2: Multicentric Carcinoma in a 51-year-old Woman with Two Palpable Masses in the Left Breast
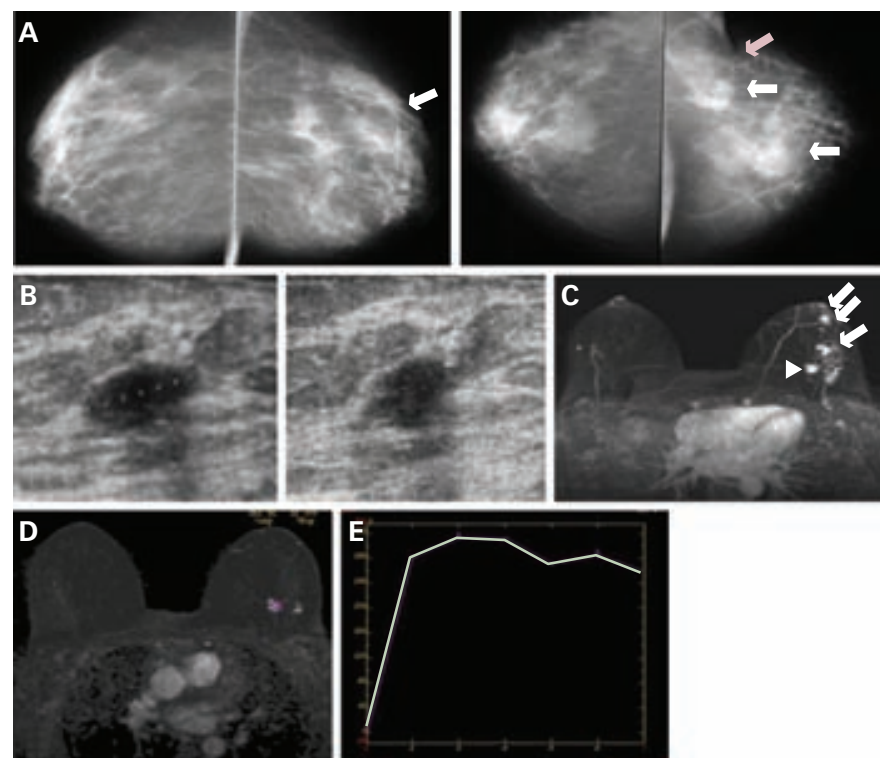

A: Mammography, mediolateral oblique and craniocaudal projections of both breasts show fibroglandular tissue located fundamentally in the upper external quadrants, an area of tissue asymmetry (white arrows) and a calcification of benign aspect (pink arrow) in the left external quadrant. B: Ultrasound scan centred on the region in which the patient noticed the increase of density shows a mass with suspicious characteristics: polylobulated, with irregular margins and acoustic posterior shadow. C-E: Enhanced magnetic resonance imaging (MRI), maximum-intensity projection (MIP) reconstruction (C): several dominant lesions in left external quadrants not detected at ultrasound are shown. Linear/micronodular enhancement (arrowhead) of the surrounding parenchyma and a growth of vascularity are associated (arrows). A subtraction image of the larger posterio lesions (D) shows a type III enhancement curve (E). Biopsy showed multifocal infiltrative ductal carcinoma (G3) with foci of micropapillar and colloid infiltration, an intramammary metastatic lymphatic nodule and extent component of intraductal carcinoma (high nuclear grade). Metastasis in seven of 10 axillary lymphatic nodules. The treatment decided on mastectomy with lymphadenectomy, posterior reconstruction and right breast reduction.

Figure 3: Occult Multicentric Carcinoma Detected by Magnetic Resonance Imaging

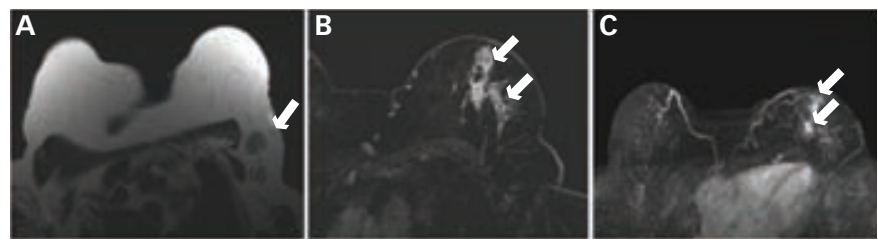

Magnetic resonance imaging (MRI) of a 69-year-old woman with a left palpable axillary lymph node and negative mammogram and sonogram (not shown). A: Pre-gadolinium T1-weighted sequence shows enlarged left axillary lymph nodes (arrow). B and C: Post-gadolinium MRI (B) and maximum-intensity projection (MIP) reconstruction (C) show enhancing retroareolar masses (arrows) in the left breast with micronodular and linear parenchymatous enhancement. The histology demonstrated poor differentiated (G3) infiltrative ductal carcinoma with extensive intraductal component and axillary metastases in seven of 17 lymphatic nodules. The treatment decided on was tumourectomy and lymphadenectomy.

only glandular asymmetry, and is more sensitive than mammography for invasive cancer in non-fatty breasts. ${ }^{7}$ For this reason, ultrasound with mammogram and medical examination is indispensable for the diagnosis of benign and malignant breast lesions.

The signs of malignancy in sonography are spiculated nodule, angular margins, marked hypoechogenicity, posterior shadow, greater height than width of the nodule, dotted calcifications, ductal extension, ramified aspect and microlobulated contours. The classic echographic appearance of the invasive carcinoma is a hypoechoic mass with internal echoes, heterogeneous, with irregular margins and acoustic shadow. ${ }^{8}$ 
Figure 4: Chemotherapy Response Evaluation with Magnetic Resonance Imaging

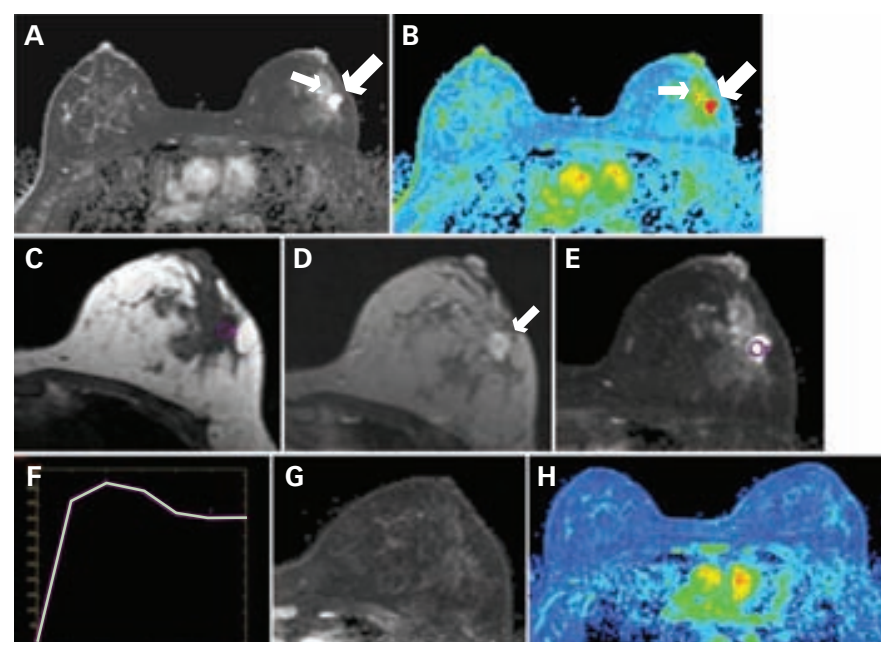

A-F: Pre-chemotherapy magnetic resonance imaging (MRI) in a 36-year-old woman with a palpable lesion in the left breast that has grown. Maximum-intensity projection (MIP) axial reconstructions and gray-scale (A) and colour-coded maps (B) show a spiculated enhancing mass (large arrow) in the upper lateral quadrant of the left breast. Closer to the skin, another smaller enhancing lesion (small arrow) is seen. Important enhancement in the surrounding parenchyma is also noted. Fine-needle aspiration cytology (FNAC) was positive for carcinoma. Unenhanced (C), post-gadolinium (D) and subtraction images (E) of the left breast show an irregular mass (arrow) with peripheral enhancement and a type III enhancement curve in the dynamic study (F). Biopsy (pre-chemotherapy): poorly differentiated infiltrating ductal carcinoma (G3). G-H: MRI post-chemotherapy. Postgadolinium axial image: grey-scale (G) and colour-coded maps (H). No areas of pathological enhancement are noted. No suspicious images are observed. Histology: no tumour found in the piece of quadrantectomy post-chemotherapy. There were eight negative lymph nodes and partial fibrosis in two other lymphatic nodules, which could correspond to complete regression of metastatic foci. The treatment decided on was quadrantectomy with lymphadenectomy and radiotherapy.

Stavros et al., applying strict echographic criteria of benignancy obtained a sensitivity of ultrasound for depicting malignancy of 98.4\%. ${ }^{\circ}$ Some authors recommend bilateral whole breast ultrasound in pre-operative evaluation of patients with breast cancer. 9.10

\section{Magnetic Resonance Imaging Indications}

Breast magnetic resonance imaging (MRI) is the most sensitive additional diagnostic method, with sensitivity values ranging between 89 and $100 \%,{ }^{3,11}$ and is developing widespread acceptance as a complementary procedure to mammography. ${ }^{12} \mathrm{MRI}$ is indicated for chemotherapy response evaluation ${ }^{13,14}$ (see Figure 4), high-risk patient screening (still controversial), primary tumour detection in patients with metastatic lymph nodes of unknown origin and detection of breast implant rupture..$^{15}$ There are different opinions regarding the accuracy of MRI for the evaluation of chemotherapy response: some authors refer to overestimation of the residual tumour ${ }^{13,14}$ and others to underestimation and false-negatives. ${ }^{16,17}$ There is controversy about the use of MRI for the evaluation of microcalcifications: some studies advise against MRI in this situation, while others propose the technique to avoid unnecessary biopsies. ${ }^{18}$ In any case, MRI is useful for pre-surgical staging of a possible ductal carcinoma in situ. ${ }^{15}$

Breast MRI has similar sensitivity and specificity to galactography and a higher negative predictive value for the detection of intraductal lesions in women with nipple discharge, with the advantage that it is a non-invasive technique. ${ }^{19}$

Special cases are those consisting of residual tumour after resection, with positive macroscopic tumour margins or no evidence of cancer in the surgical piece (excluding false-positives of biopsy). In these patients, MRI is useful for locating the tumour. When there is malignancy after resection of a supposed benign lesion, MRI should be performed for re-staging and detection of additional foci or multicentric or contralateral cancer. Post-surgical MRI should always be performed in the first two weeks after the excision, because later granulation tissue can cause false-positives. ${ }^{15}$

Concerning lesions of uncertain malignant potential or B3 (Nottingham classification after percutaneous biopsy), which include radial scars, papillary lesions, lobular carcinoma in situ, atypical lobular hyperplasia, flat epithelial atypia and atypical ductal hyperplasia, there is no scientific evidence concerning the use of MRI for the detection of concomitant malignant lesions. ${ }^{20}$ The American Cancer society does not recommend follow-up with MRI in these patients; ${ }^{21}$ nevertheless other guides recommend performing breast MRI annually in patients with lobular carcinoma in situ.22

The utility of MRI for screening high-risk patients and for the staging of breast cancer, and its impact on clinical management, will be discussed later.

\section{Technique}

Multiple breast MRI protocols are described in the literature. Most of the studies are carried out with a high-field magnet (1.0-1.5T) using specific breast surface superficial coils. In general, the study includes both breasts, especially in pre-surgical staging and post-surgical evaluation. Patients are laid down in the prone position and paramagnetic intravenous contrast is almost always administered using an injection pump $(0.1-0.2 \mathrm{mmol} / \mathrm{kg}$ at a rate of $2 \mathrm{ml} / \mathrm{second}$, followed by $15-20 \mathrm{ml}$ of saline solution at the same rate). The acquisition plane is axial or coronal for bilateral studies, although sagittal images can also be useful..$^{23}$

Our protocol includes axial and coronal $\mathrm{T}_{2}$-weighted sequences with and without fat saturation (FS), which are particularly useful for the characterisation of certain lesions (cysts) and to evaluate post-surgical changes. Subsequently, dynamic $\mathrm{T}_{1}$-weighted sequences with high temporary and spatial resolution are acquired in the axial plane before and after the administration of a gadolinium chelate. These last sequences are the essential base of breast MRI. Fat suppression images can also be obtained by post-processing (subtraction) techniques. ${ }^{23}$ We administer $0.1 \mathrm{mmol} / \mathrm{kg}$ of intravenous gadolinium chelate and use dedicated breast surface coils.

Post-processing of the images is performed in a workstation by an expert radiologist, who also carries out the subtraction and quantification of the enhancement. The most representative reformatted images are sent to the picture archiving and communication system (PACS) and a hard copy is attached to the report for the patient.

\section{Findings and Interpretation}

Breast MRI should be read by a suitable expert, which is not the case at the majority of institutions. Images should be evaluated in a workstation as mentioned, completing post-processing that includes the subtraction and quantification of the enhancement. ${ }^{23} \mathrm{~A}$ great variety of morphological and dynamic criteria is used to evaluate suspicious findings. The American College of Radiology (ACR) has developed the lexicon BI-RADS-MRI to homogenise the language used 
in the reports and avoid confusing terms. The enhancement of injuries is classified into three categories: foci, masses and non-mass-like enhancement. In each one, some characteristics that determine the degree of suspicion of malignancy should be analysed. ${ }^{23-26}$

Two methods can be used to evaluate the enhancement: quantitative and qualitative. Quantitative methods evaluate the enhancement by means of formulae to calculate the rates of enhancement immediately after the administration of contrast. Qualitative methods analyse the morphology of the curves of enhancement. According to this, three types of curve are described. Type I curves consist of a persistent and progressive enhancement. Type $\|$ is an initial enhancement that persists and is stable in the delayed phases; this curve is also known as a 'plateau' pattern. Type III refers to an initial important enhancement with posterior quick washout ${ }^{23-26}$ (see Figures 1-3).

To establish the probability of malignancy, integration of the morphological characteristics and kinetic information of the enhancement should be carried out. Imaging features associated with malignancy are similar to those of conventional mammograms irregular form or margins, spiculated borders; but there are also some that are specific to enhanced MRI - ring enhancement and type II or III curves. ${ }^{23-26}$

\section{Role of Breast Magnetic Resonance Imaging in the Approach to Clinical Management and Treatment}

The approach to breast cancer treatment has changed drastically in recent decades. Improvements in detection of the illness and the knowledge that the majority of treatment failures occur due to visceral or systemic recurrence has enabled a change in breast cancer management. Conservative procedures have increased and the survival rate has improved.

The therapeutic options basically depend on local staging of the primary tumour. In invasive or non-invasive operable carcinomas, the treatment will be based on surgical resection, radiotherapy or both. For systemic breast cancers, chemotherapy, hormone therapy or biologic therapies are recommended. The complete staging and histological characteristics of breast cancer are fundamental for the choice of best treatment. Cancer extent, lymph-node infiltration, hormonal receptor presence, histology of the tumour, HER2/neu gene expression, metastatic disease and the physical condition of the patient will establish the tumour, node, metastasis (TNM) staging and will help in the estimation of risk and prediction of treatment response. ${ }^{1,27,28}$

It has been demonstrated that breast MRI is the most sensitive additional diagnostic method for diagnosing all tumour types, with sensitivity values ranging between 89 and 100\%,3,11,27,29,30 Some authors report that accurate pre-surgical staging provided by MRI leads to a reduction in recurrence and patients with positive tumour margins:; however, other experts advise that there is little high-quality evidence at present to support the routine use of pre-operative MRI, 33,34 and in some cases MRI can be harmful due to false-positives adding unnecessary biopsies and surgery. ${ }^{34,35}$

A different concern is the level of accuracy for predicting tumour size, which is very important for surgical planning. Mammography and ultrasound often underestimate tumour size, which can result in incomplete resection. ${ }^{36,11}$ In this area, MRI plays an essential role in surgical planning and therapy choice, because it shows more precisely than mammography the local extent of breast cancer, tumour size, carcinoma location and chest wall infiltration. 11,13,27,37-48 Even more importantly, some carcinomas or foci are seen only on breast MRI (see Figure 3). ${ }^{30,31,38,39,41,42,49-51}$

The majority of breast imaging experts reinforce the concept that the essential value of MRI in the pre-surgical staging of breast cancer is its high sensitivity for the detection of additional foci of cancer that are non-detectable with other imaging techniques, which implies a new therapeutic approach for these patients. The capacity of breast MRI for the detection of additional carcinoma foci and occult carcinomas has been demonstrated in several studies. ${ }^{3,11,13,27,29-31,37-53}$ For this reason, to minimise potential local failures, ${ }^{42,51}$ breast MRI is recommended before partial breast irradiation and should be integrated into the work-up of patients considered for breast-conserving treatment. ${ }^{54} \mathrm{MRI}$ is even considered for pre-operative evaluation of all newly diagnosed breast cancer patients. . $^{27,13,38,43,45,46}$ The benefits of this proposal theoretically are improvements to the selection process for breast-conserving surgery, a decrease in the number of surgical procedures required to obtain negative lumpectomy margins and the synchronous detection of contralateral cancers. Nevertheless, the proposal is controversial: Houssami et al. noted that there are no data from prospective randomised trials to support these assertions. ${ }^{33,35}$

What is confirmed is that in many cases pre-surgical MRI modifies the treatment of breast cancer due to the visualisation of additional foci. Fischer et al. ${ }^{55}$ scored a change in surgical planning in 66 of 336 women (19.6\%) due to the detection in breast MRI of unsuspected multifocal cancer in $8.9 \%$, multicentric cancer in $7.1 \%$ and contralateral carcinoma in $4.4 \%$ of patients. Tillman et al. ${ }^{37}$ found a modification of clinical management in $20 \%$ of women with early-stage breast cancer due to the results of breast MRI. The changes were favourable in $11 \%$ of patients, with multiple foci detected, better localisation of tumours and characterisation of secondary tumours that were doubtful findings with other techniques. The modification was uncertain in $2 \%$, led to unnecessary biopsies in $5 \%$ and was negative in $1 \%$ of women who underwent mastectomy when they could have been treated with conservative surgery. Martinez et al. ${ }^{48}$ refer to the detection of additional foci of cancer in $20 \%$, which led to a change in the type of surgery in $14.4 \%$ of patients. These changes were lumpectomy to extended resection or quadrantectomy due to multifocal carcinoma, conservative surgery to mastectomy due to a vast multifocal carcinoma or multicentric cancer, or change of unilateral surgery for bilateral intervention due to synchronous bilateral carcinoma, all of them detected in pre-surgical MRI.

Regarding the utility of MRI for screening in high-risk patients, MRI is especially indicated in cases of heterogeneous or extremely dense breasts due to the limitations of mammography in this group of patients. ${ }^{3,29,52,53}$ Sardanelli recommends that patients with a potentially higher anticipated benefit from pre-operative MRI can be identified as those with mammographically dense breasts, with unilateral multifocal/multicentric cancer or a synchronous bilateral cancer already diagnosed at mammography and sonography, with a lobular invasive cancer, at high risk of breast cancer, with a cancer that shows a discrepancy in size of $>1 \mathrm{~cm}$ between mammography and sonography, or under consideration for partial breast irradiation. More limited evidence exists in favour of MRI for evaluating candidates for total skin-sparing mastectomy or for patients with 
Figure 5: False-positive Finding on Enhanced Magnetic Resonance Imaging

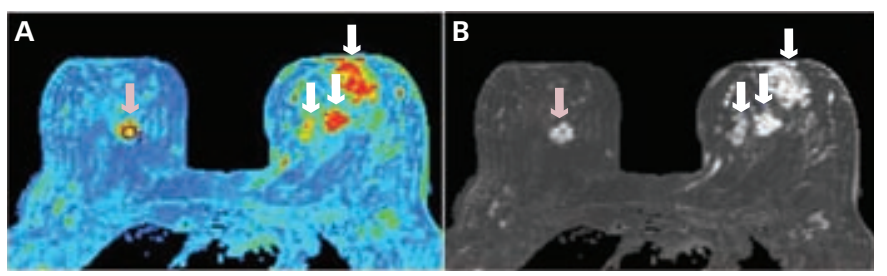

Magnetic resonance imaging (MRI) of a 49-year-old patient with palpable nodule in the left breast and a suspicious lesion in the mammogram (not shown). A and B. Post-gadolinium MRI, maximum-intensity projection (MIP) axial reconstruction, grey-scale (A) and colourcoded maps (B), show multiple lesions in the left breast (white arrows) and a suspicious microlobulated enhancing mass in the right (pink arrow), suggestive of bilateral carcinoma. The histology was locally advanced multicentric carcinoma in the left breast (poorly differentiated infiltrative ductal carcinoma) and fibroadenoma in the right breast. The treatment decided on was left mastectomy with lymphadenectomy

Paget's disease. Irrespective of whether the clinical team routinely uses pre-operative MRI, women who are newly diagnosed with breast cancer should always be informed of the potential risks and benefits of pre-operative MRI, and radiologists should always remember that the results of MRI should be interpreted by taking into account clinical breast examination, mammography and sonography and should be verified by percutaneous biopsy. ${ }^{47}$ According to this, MR guidance for needle biopsy and pre-surgical localisation should be available or potentially accessible if pre-operative MRI is to be implemented, as lesions detected only on MRI are not infrequent. ${ }^{47,56,57}$

The most important disadvantage of breast MRI and the most controversial point in its use for breast cancer staging is its low specificity, which is between 50 and $70 \%$ in the majority of the literature, ${ }^{11,23,27,29,37}$ but is extremely variable (37-97\%) depending on the technique used, parameters and interpretation criteria. ${ }^{3,37,23}$ An unexpected enhancement of lesions occurs on 16-29\% of breast MRI. ${ }^{23}$ Normal breast parenchyma can enhance symmetrically or with a nodular pattern, particularly in pre-menopausal women, which limits the role of MRI by causing false-positives (see Figure 5). In order to decrease the probability of detecting false-positives, MRI should be performed during the first few days of the menstrual cycle (days five to fifteen), because the parenchymatous enhancement is less intense in this period. ${ }^{3,23,24}$ Several lesions can simulate breast cancer in MRI, including benign injuries such as fibroadenomas (see Figure 5), papillomas, lymphatic nodules, high-risk injuries such as lobar carcinoma in situ, atypical ductal hyperplasia, atypical lobular hyperplasia and a miscellaneous group that includes fibrocystic changes, adenosis, sclerosis, ductal hyperplasia and fibrosis..$^{23,24}$ Therefore, it is extremely important to always obtain a biopsy before changing the treatment approach after the detection of a lesion in a pre-surgical staging MRI. $3,24,29,56$

Other limitations of breast MRI are lower sensitivity for ductal carcinoma in situ ${ }^{50}$ than for invasive tumours (which is extremely variable between 60 and $90 \%,{ }^{27} 50$ and $80 \%{ }^{3}$ and 40 and $100 \%{ }^{24}$ in different series), high cost, limited availability ${ }^{50}$ and, in particular, the lack of availability of MRI-compatible biopsy equipment. ${ }^{3,4,11,47,54,55}$

\section{Summary}

Triple Assessment versus Mammography and Ultrasound in Breast Cancer Evaluation

The main advantages of breast MRI for the evaluation of breast cancer are: greater accuracy than mammography and ultrasound to determine the local extent, tumour size and location, ${ }^{11,13,27,37-48}$ which means a better indication of the most suitable therapy, better evaluation of heterogeneous or extremely dense breasts, ${ }^{3,29,52,53}$ high sensitivity for the detection of additional foci of occult cancer, 3,11,13,27,29${ }^{31,37-53}$ which imparts a new approach to possible therapies (see Figures 1-3) and its capacity to evaluate post-chemotherapy changes ${ }^{13,14,16,17}$ (see Figure 4). The most important disadvantage is its low specificity (50-70\%), 112,23,27,29,37 which means that in breast cancer staging all suspicious MRI-detected lesions must always be biopsied before changing therapy. ${ }^{3,24,29,56}$ In any case, there are subgroups of patients who profit the most from MRI staging: women with ductal carcinoma in situ, those with infiltrating lobular carcinoma, patients who are going to be treated with neoadjuvant chemotherapy, patients with Paget's disease or those with dense breasts who are going to undergo modified radical mastectomy. ${ }^{15}$

Radiologists should never forget that in breast cancer screening the combination of mammography, ultrasonography, clinical examination and MRI is more sensitive than any other individual test or combination of tests. ' A clear understanding of the valid indications and selection criteria for using breast MRI appropriately ${ }^{12}$ is fundamental, and clear communication to patients about the limits of MRI should be established due to the possibility of false-positive findings. ${ }^{58}$

\section{Future Directions}

The impact of the use of breast MRI on survival and recurrence rates in breast cancer is not well established. Some authors report that the multicentric foci of multifocal carcinomas detected on MRI could have been properly treated with radiotherapy and chemotherapy after surgery, ${ }^{54,59-61}$ which means that all additional foci diagnosed with MRI have no real impact on survival and recurrence rates, with an increased false-positive rate and unnecessary surgery. ${ }^{34,35}$ In a recently published meta-analysis, ${ }^{35}$ the percentage of additional foci detected in MRI and the percentage of patients with a change in surgical planning due to those findings was $7.8-33.3 \%$. A modified radical mastectomy was executed instead of conservative surgery in $8.1 \%$ of patients with malignant lesions and $1.1 \%$ of patients with false-positives. Extension of conservative surgery was carried out in $11.3 \%$ of patients with additional carcinoma foci and $5.3 \%$ of patients with benign lesions. The same authors concluded in a posterior study that MRI adds unnecessary surgery and does not demonstrate a better survival rate..$^{33}$ An important fact in all these discussions is that in breast divisions that integrate all imaging techniques (mammography, ultrasonography and MRI) and interventional procedures, the rate of incorrect change in the therapy is low, at $3.8 \%{ }^{62}$ This indicates that integration of MRI into the work-up as an additional imaging technique in pre-surgical staging of breast cancer is mostly beneficial.

Concerning the re-operation rate, Pengel et al. ${ }^{32}$ recently published the first randomised clinical trial to compare patients staged with MRI versus patients who did not undergo MRI for staging. In the first group the re-operation rate was $13.8 \%$, and in the second, $19.1 \%$. The authors concluded that MRI does not significantly affect the re-operation rate but decreases ductal carcinoma in situ with a positive margin rate. The most recent publication, which covered the Comparative Effectiveness of MRI in Breast Cancer study (COMICE), a randomised controlled trial, confirmed that the addition of MRI to conventional triple assessment is not significantly associated with a reduced re-operation rate, so may be 
unnecessary, ${ }^{63}$ but did not fully answer whether pre-operative breast MRI adds benefit because recurrence and overall survival rates were not examined.

Specifically in terms of the recurrence rate, Fisher et al. ${ }^{27}$ obtained better results: $1.2 \%$ recurrences in patients with a pre-surgical MRI versus $6.8 \%$ in women without it. The rate of undetected contralateral carcinomas in the follow-up was $1.7 \%$ in women with pre-surgical MRI versus $4 \%$ of women without MRI. Nevertheless, Drew et al. ${ }^{64}$ did not see any impact of pre-surgical MRI on the local recurrence rate, and Solin et al. ${ }^{59}$ did not observe any difference in the incidence of contralateral carcinoma in patients with or without pre-surgical MRI. All these studies indicate that there is still no consensus about the impact of MRI on recurrence rate. On the other hand, the new techniques of minimally invasive treatment, such as radiofrequency, microwaves, high-intensity focused ultrasound and partial breast radiotherapy instead of radiotherapy of the whole breast, will require a very accurate technique for staging - and that technique will certainly be MRI. ${ }^{15}$

\section{Conclusion}

Breast MRI is the most sensitive imaging modality for the detection of invasive carcinoma ${ }^{3,11,27,2,37}$ and must be evaluated together with the remainder of the conventional imaging techniques. ${ }^{7.62}$ It plays an essential role in the detection of additional tumour foci, especially in young women with a family history of breast cancer, those with dense breasts and women with primary lobular carcinoma., ${ }^{3,1,27,29,37}$ Findings in MRI can modify the surgical approach in these patients, ${ }^{35,37,48,55,62}$ so radiologists should be aware of false-positives that can lead to unnecessary biopsies or surgeries and more aggressive treatments. The impact of pre-surgical staging MRI on the rate of re-operation, recurrences and survival, remains controversial.
1. Ellis $I O$, Schnitt SJ, Sastre-Garau $X$, et al., Invasive breast carcinoma. In: Tavassoli FA, Deville P (eds), World Health Organization Classification of Tumours. Pathology and Genetics of Tumours of the Breast and Female Genital Organs, 1st edn, Lyon: IARC Press, 2003

2. Mosquera Oses J, Controversies in breast cancer screening, Radiología, 2010;52:3-6.

3. Sardanelli F, Giuseppetti GM, Panizza P, et al., Sensitivity of MRI versus mammography for detecting foci of multifocal, multicentric breast cancer in fatty and dense breasts using the whole-breast pathologic examination as a gold standard, the whole-breast pathologic exam.

4. American College of Radiology (ACR). ACR BI-RADS ${ }^{\circledR}$ Mammography, 4th edn. In: ACR Breast Imaging Reporting and Data System, Breast Imaging Atlas, Reston, VA: American Colleg of Radiology, 2003

5. Martín Díez F, Controversies in breast sonography, Radiología, 2010;52:22-5

6. Stavros AT, Thickman D, Rapp CL, et al., Solid breast nodules: use of sonography to distinguish between benign and malignant lesions, Radiology, 1995:196:123-34.

7. Berg WA, Gutierrez L, NessAiver MS, et al., Diagnostic accuracy of mammography, clinical examination, US and MR Imaging in preoperative assessment of breast cancer, Radiology, 2004;233:830-49.

8. American College of Radiology (ACR). ACR BI-RADS ${ }^{\circledR}$ Ultrasound, 4th edn. In: ACR Breast Imaging Reporting and Data System, Breast Imaging Atlas, Reston, VA: American College of Radiology, 2003

9. Berg WA, Gilbreath PL, Multifocal and multicentric cancer: whole-breast US in preoperative evaluation, Radiology, 2000;214:59-66

10. Moon WK, Noh DY, Im JG, Multifocal, multicentric, and contralateral breast cancers: bilateral whole-breast US in the preoperative evaluation of patients, Radiology, 2002;224:569-76.

1. Caramella T, Chapellier C, Ettore F, et al., Value of MRI in the surgical planning of invasive lobular breast carcinoma: a prospective and retrospective study of 57 cases. Comparison with physical examination, conventional imaging and histology, Clin Imaging, 2007;31:155-61.

12. Lalonde L, David J, Trop I, Magnetic resonance imaging of the breast: current indications, Can Assoc Radiol J, 2005:56:301-8.

13. Balu-Maestro $C$, Chapellier $C$, Bleuse $A$, et al., Imaging in evaluation of response to neoadjuvant breast cancer treatment benefits of MRI, Breast Cancer Res Treat, 2002;72:145-52.

14. Kim HJ, Im YH, Han BK, et al., Accuracy of MRI for estimating residual tumour size after neoadjuvant chemotherapy in locally advanced breast cancer: relation to response pattern on MRI, Acta Oncol, 2007;46:996-1003

15. Camps Herrero J, Controversies in breast MRI, Radiología, 2010:52:26.

16. Vénat-Bouvet $L$, Desfougères $M$, Aubard $Y$, et al., MR evaluation of primary chemotherapy response in breast caluation of primary chemotherapy r

17. Kwong MS, Chung GG, Horvath $\mathrm{L}$, et al., Postchemotherapy MRI overestimates residual disease compared with histopathology in responders to neoadjuvant therapy for locally advanced breast cancer, Cancer J, 2006;12:212-21.

18. Kneeshaw PJ, Lowry M, Manton D, et al., Differentiation of benign from malignant breast disease associated with screening detected microcalcifications using dynamic contrast enhanced magnetic resonance imaging, Breast, 2006;15:29-38.

19 Morrogh M, Morris EA, Liberman L, et al., The predictive value of ductography and magnetic resonance imaging in the management of nipple discharge, Ann Surg Oncol, 2007:14:3369-77.

20. Linda A, Zuiani C, Bazzocchi M, et al., Borderline breas lesions diagnosed at core needle biopsy: can magnetic resonance mammography rule out associated malignancy?
Preliminary results based on 79 surgically excised lesions, Breast, 2008:17:125-31.

21. Saslow D, Boetes C, Burke W, et al., American Cancer Society guidelines for breast screening with MRI as an adjunct to mammography, CA Cancer I Clin, 2007;57:75-89.

22. The National Comprehensive Cancer Network (NCCN), Breast Cancer Screening and Diagnosis, Practice Guidelines in Oncology v.2.2009.

23. Kuhl C, The current status of breast MR imaging. Part I. Choice of technique, image interpretation, diagnostic accuracy and transfer to clinical practice, Radiology, 2007:244:356-78.

24. Macura KJ, Ouwerkerk R, Jacobs MA, Bluemke DA, Patterns of enhancement on breast MR Images: Interpretation and imaging pitfalls, Radiographics, 2006;26:1719-34.

25. Berg WA, Gutierrez L, NessAiver MS, et al., Diagnostic accuracy of mammography, clinical examination, US and M imaging in preoperative assessment of breast cancer, Radiology, 2004;233:830-49.

26. American College of Radiology (ACR), ACR BI-RADS ${ }^{\circledR}$ Magnetic Resonance Imaging, 4th edn. In: ACR Breast Imaging Reporting and Data System, Breast Imaging Atlas, Reston, VA: American College of Radiology, 2003.

27. Fischer $U$, Zachariae $\mathrm{O}$, Baum $F$, et al., The influence of preoperative MRI of the breasts on recurrence rate in patients with breast cancer, Eur Radiol, 2004;14:1725-31.

28. Solin LJ, Breast conservation treatment with radiation: an ongoing success story, I Clin Oncol, 2010;28(5):709-11.

29. Van Goethem M, Schelfout K, Dijckmans L, et al., MR mammography in the pre-operative staging of breast cance in patients with dense breast tissue: comparison with mammography and ultrasound, Eur Radiol, 2004;14:809-16.

30. Mann RM, The effectiveness of MR imaging in the assessment of invasive lobular carcinoma of the breast, Magn Reson Imaging Clin North Am, 2010;18(2):259-76, ix

31. Lim HI, Choi JH, Yang JH, et al., Does pre-operative breast magnetic resonance imaging in addition to mammography and breast ultrasonography change the operative management of breast carcinoma?, Breast Cancer Res Treat, 2010;119:163-7.

32. Pengel KE, Loo CE, Teertstra HJ, et al., The impact of preoperative MRI on breast-conserving surgery of invasive cancer: a comparative cohort study, Breast Cancer Res Treat, 2009;116(1):161-9

33. Houssami N, Hayes DF, Review of preoperative magnetic resonance imaging (MRI) in breast cancer: should MRI be performed on all women with newly diagnosed, early stage breast cancer? CA Cancer I Clin, 2009;59:290-302.

34. Solin L, Counterview: pre-operative breast MRI (magnetic resonance imaging) is not recommended for all patients with newly diagnosed breast cancer, Breast, 2010;19:7-9.

35. Houssami N, Ciatto S, Macaskill P, et al. Accuracy and surgical impact of magnetic resonance imaging in breast cancer staging: systematic review and meta-analysis in detection of multifocal and multicentric cancer, I Clin Oncol, 2008:26:3248-58

36. Mann RM, Veltman J, Barentsz JO, et al., The value of MR compared to mammography in the assessment of tumour extent in invasive lobular carcinoma of the breast, Eur I Surg oncol, 2008;34:135-42.

37. Tillman GF, Orel SG, Schnall MD, et al., Effect of breast magnetic resonance imaging on the clinical management of women with early-stage breast carcinoma, J Clin Oncol 2002;20:3413-23.

38. Schelfout K, Van Goethem M, Kersschot E, et al., Contrastenhanced MR imaging of breast lesions and effect on treatment, Eur I Surg Oncol, 2004:30:501-7.

39. Kneeshaw PJ, Turnbull LW, Smith A, Drew PJ, Dynamic contrast enhanced magnetic resonance imaging aids the surgical management of invasive lobular breast cancer, Eur I Surg Oncol, 2003;29:32-7.

40. Bilimoria KY, Cambic A, Hansen NM, Bethke KP, Evaluating the impact of preoperative breast magnetic resonance imaging on the surgical management of newly diagnosed breast cancers, Arch Surg, 2007;142:441-5, discussion 445-7.

41. Drew PJ, Chatterjee S, Turnbull LW, et al., Dynamic contrast enhanced magnetic resonance imaging of the breast is superior to triple assessment for the pre-operative detection of multifocal breast cancer, Ann Surg Oncol, 1999;6:599-603.

42. Al-Hallaq HA, Mell LK, Bradley JA, et al., Magnetic resonance imaging identifies multifocal and multicentric disease in breast cancer patients who are eligible for partial breast irradiation, cancer, 2008:113:2408-14.

43. Hollingsworth $A B$, Stough RG, O'Dell CA, Brekke CE, Breast magnetic resonance imaging for preoperative locoregional staging, Am I Surg, 2008;196:389-97.

44. Siegmann KC, Baur A, Vogel U, et al., Risk-benefit analysis of preoperative breast MRI in patients with primary breast cancer, Clin Radiol, 2009;64:403-13.

45. Pediconi F, Catalano C, Padula S, et al., Contrast-enhanced magnetic resonance mammography: does it affect surgical decision-making in patients with breast cancer?, Breast Cancer Res Treat, 2007;106:65-74.

46. Pediconi $F$, Catalano $C$, Roselli A, et al., contrast-enhanced MR mammography for evaluation of the contralateral breast in patients with diagnosed unilateral breast cancer or highrisk lesions, Radiology, 2007;243:670-80.

47. Sardanelli F, Overview of the role of pre-operative breast MR in the absence of evidence on patient outcomes, Breast, 2010;19:3-6.

48. Martínez V, Carreira MC, Pérez Y, et al., Effect of preoperative magnetic resonance imaging on the surgical treatment of breast carcinoma, Prog Obstet Ginecol, 2007;50:682-8.

49. Mann RM, Hoogeveen YL, Blickman JG, Boetes C, MR compared to conventional diagnostic work-up in the detection and evaluation of invasive lobular carcinoma of the breast: a review of existing literature, Breast Cancer Res Treat, 2008;107:1-14

50. Gundry KR, The application of breast MRI in staging and screening for breast cancer, Oncology (Williston Park), 2005;19:159-69, discussion 170, 173-4, 177.

51. Tendulkar RD, Chellman-Jeffers M, Rybicki LA, et al., Preoperative breast magnetic resonance imaging in early breast cancer: implications for partial breast irradiation, Cancer, 2009;115:1621-30.

52. Pediconi $F$, Catalano $C$, Roselli $A$, et al.., The challenge of imaging dense breast parenchyma: is magnetic resonance mammography the technique of choice? A comparative study with $\mathrm{x}$-ray mammography and whole-breast ultrasound, Invest Radiol, 2009;44:412-21.

53. Olivas-Maguregui $S$, Villaseñor-Navarro $Y$, Ferrari-Carballo $T$, et al., Importance of the preoperative evaluation of multifocal and multicentric breast cancer with magnetic resonance imaging in women with dense parenchyma, Rev Invest Clin, 2008;60:382-9.

54. Kuhl C, Kuhn W, Braun M, Schild H, Pre-operative staging of breast cancer with breast MRI: one step forward, two steps back?, Breast, 2007;16:S34-44.

55. Fischer U, Baum F, Luftner-Nagel S, Preoperative MR imaging in patients with breast cancer: preoperative staging, effects on recurrence rates, and outcome analysis, Magn Reson Imaging Clin N Am, 2006;14:351-62, vi.

56. Kuhl CK, Braun M, Magnetic resonance imaging in preoperative staging for breast cancer: pros and contras, Radiologe, 2008;48:358-66.

57. Lehman $\mathrm{CD}$, DeMartini $\mathrm{W}$, Anderson $\mathrm{BO}$, Edge $\mathrm{SB}$, Indications for breast MRI in the patient with newly diagnosed breast cancer, I Nat/ Compr Canc Netw, 2009:7:193-201.

58. McCaffery KJ, Jansen J, Pre-operative MRI for women with newly diagnosed breast cancer: perspectives on clinician and patient decision-making when evidence is uncertain, Breast, 2010;19:10-2.

59. Solin LJ, Orel SG, Hwang WT, et al., Relationship of breast 
magnetic resonance imaging to outcome after breastconservation treatment with radiation for women with earlystage invasive breast carcinoma or ductal carcinoma in situ, I Clin Oncol, 2008;26:386-91.

60. Fisher B, Anderson S, Bryant J, et al., Twenty-year follow-up of a randomized trial comparing total mastectomy, lumpectomy and lumpectomy plus irradiation for the treatment of invasive breast cancer, N Engl J Med, 2002;347:1233-41.

61. Veronesi U, Cascinelli N, Mariani L, et al., Twenty-year followup of a randomized study comparing breast-conserving surgery with radical mastectomy for early breast cancer, N Eng/ J Med, 2002;17;347:1227-32.

62. Camps J, Sentis M, Ricart V, et al., Utilidad de la resonancia magnética en la evaluación local del cáncer de mama: impacto en el cambio de actitud terapéutica en una serie prospectiva de 338 pacientes, Rev Senología Patol Mam, 2007;20:53-66.

63. Turnbull L, Brown S, Harvey I, et al., Comparative effectiveness of MRI in breast cancer (COMICE) trial: a randomised controlled trial, Lancet, 2010;375:563-71. 64. Drew $P$, Turnbull $L$, Harvey I, et al., MR imaging in breas 